\title{
Inception of the Modern Public Health System in China and Perspectives for Effective Control of Emerging Infectious Diseases: In Commemoration of the 140th Anniversary of the Birth of the Plague Fighter Dr. Wu Lien-Teh
}

\author{
Qingmeng Zhang ${ }^{1,2} \cdot$ Niaz Ahmed $^{2} \cdot$ George F. Gao ${ }^{1,3,4} \cdot$ Fengmin Zhang $^{1,2}$ (I)
}

Received: 30 January 2020 / Accepted: 28 June 2020/Published online: 3 August 2020

(c) Wuhan Institute of Virology, CAS 2020

Infectious diseases pose a serious threat to human health and affect social, economic, and cultural development. Many infectious diseases, such as severe acute respiratory syndrome (SARS, 2013), Middle East respiratory syndrome (MERS, 2012 and 2013), Zika virus infection (2007, 2013 and 2015), and coronavirus disease 2019 (COVID-19, 2019), have occurred as regional or global epidemics (Reperant and Osterhaus 2017; Gao 2018; Li et al. 2020). In the past 100 years, the world has gradually established a relatively complete modern public health system. The earliest modern public health system in China was founded by the plague fighter Dr. Wu Lien-Teh during the campaign against the plague epidemic in Northeast China from 1910 to 1911 . This campaign not only played a significant part in effective control of the plague at that time, but also established a foundation for the Chinese modern public health system.

In this article, we systematically review Dr. Wu LienTeh's academic achievements and outstanding contributions in the prevention and control of the plague epidemic in northeast China and introduce the development of the earliest public health epidemic prevention system in China in order to commemorate the 140th anniversary of Dr. Wu Lien-Teh's birth. We hope that this article will provide

Fengmin Zhang

fengminzhang@ems.hrbmu.edu.cn

1 Wu Lien-Teh Institute, Harbin Medical University, Harbin 150081, China

2 Department of Microbiology, School of Basic Medical Science, Harbin Medical University, Harbin 150081, China

3 CAS Key Laboratory of Pathogenic Microbiology and Immunology, Institute of Microbiology, Chinese Academy of Sciences (CAS), Beijing 100864, China

4 Chinese Center for Disease Control and Prevention, Beijing 102206, China insights into the effective prevention and control of emerging infectious diseases as well as the current worldwide pandemic of COVID-19, facilitating the improvement and development of public health systems in China and around the globe.

\section{Etiological Investigation and Bacteriological Identification of the Plague Epidemic in the Early 20th Century}

In September 1910, the plague hit the Transbaikal region of Russia and spread to Manzhouli, a Chinese town on the border between China, Mongolia, and Russia, causing many deaths (Wu 1911; Strong 1912; Wu 1914; Wu 1959). The main symptoms exhibited by patients included headache, fever, coughing up bloody sputum, and death, all occurring within a short period. Thereafter, the disease spread fast along the Middle East Railway from Manzhouli to Harbin, where there were frequent international business contacts and a large migrant population. However, physicians and researchers from Russia, Japan, and China in Harbin were unable to confirm the cause of the disease outbreak.

In December, 1910, Dr. Wu Lien-Teh (MD, PhD; Cambridge University), the vice-director of the Imperial Army Medical College (Peiyang Medical School) in Tientsin (Tianjin, China), was appointed by the government of Qing Dynasty to deal with plague control with full authority in Harbin. On December 24, 1910, Dr. Wu LienTeh and his student Lin Chia-Swee arrived in Harbin by train with experimental reagents and a microscope and then traveled into the Fuchiatien (Fujiadian) area of Harbin to visit patients and communicate with local doctors for onsite investigation of the pestilence. He found that sporadic deaths had begun to occur in early November 1910 and 
then increased in December. Moreover, Dr. Wu Lien-Teh found that the disease, exhibiting frequent pulmonary injury similar to hemoptysis, mainly occurred in crowded and unclean areas and tended to spread among people who were in close contact with each other, thus demonstrating typical features of an infectious disease transmitted through the respiratory tract (Wu 1911; Wu 1959). On December 27 , the first autopsy of a dead patient was performed by Dr. Wu Lien-Teh, and specimens from the lungs, blood, heart, liver, spleen, and kidneys were analyzed through smear microscopy and bacterial culture. Some samples were fixed with $10 \%$ formalin for further pathological observation. Large numbers of Yersinia pestis were found in multiple organs, and $Y$. pestis was also found in the sputum of infected cases under microscopic detection (Wu 1914; Wu 1926) (Fig. 1). These findings helped to confirm that $Y$. pestis was the causative pathogen of this pestilence.

Dr. Wu Lien-Teh also found that the virulence of $Y$. pestis varied according to additives by inoculating $Y$. pestis into different types of artificial media (Wu 1918). Additionally, exposure of patient sputum containing $Y$. pestis to direct or to diffuse sunlight, cold conditions, wood and gauze surfaces, and soil showed that these environmental conditions did not kill $Y$. pestis on the smears. Similar results were found with some disinfectants and magenta dyes, whereas alcohol and basic methylene blue staining had killing effects against this microorganism (Wu and Jettmar 1926; Wu et al. 1936). Bacteriological investigation of the $Y$. pestis strain provided a foundation for the prevention and control of the plague and establishment of the pneumonic plague theory.

\section{Studies on the Natural Foci and Transmission of Pneumonic Plague and the Establishment of Pneumonic Plague Theory}

In order to investigate the natural foci of pneumonic infection, Dr. Wu Lien-Teh organized a joint expedition with his Russian colleagues to investigate wild hunting and plague epidemic conditions via field trips to the Transbaikal region of Russia, Manchouli of China, and villages of Mongolia from May to July 1911. They found that there were epidemics of plague from 1905 to 1908 and from 1910 to 1911 (Wu 1913a, b; Wu et al. 1926), establishing a

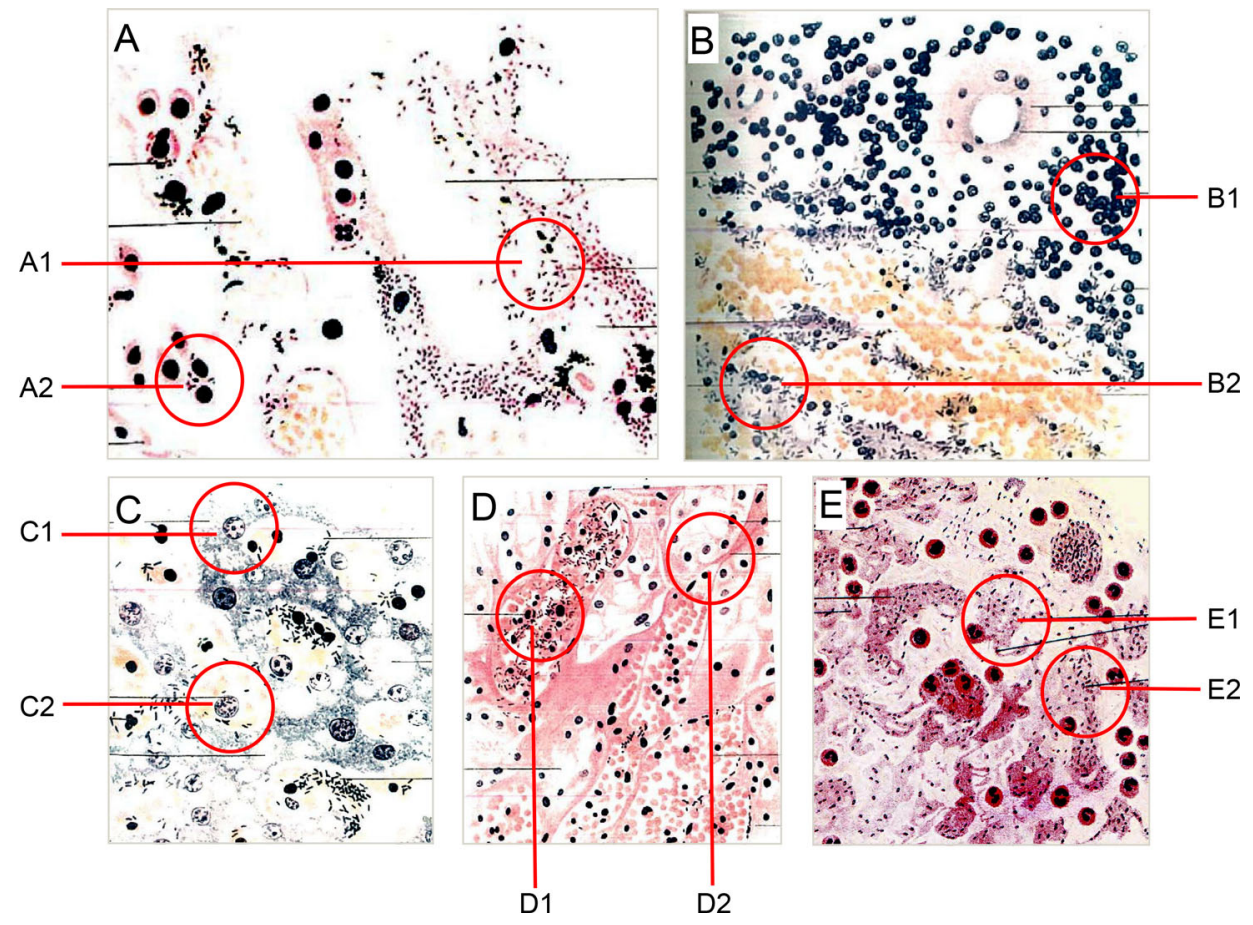

Fig. 1 Pathological and bacteriological findings of infected organs and sputum of patients with pneumonic plague. A Lung section $(300 \times$, Giemsa's stain). A1 Collapsed alveolar space. A2 Large epithelial cells containing ingested plague bacilli and carbon pigment. B Spleen section $(200 \times$, Giemsa's stain). B1 Swollen and vacuolated lymphocytes. B2 Swollen trabecula having an enormous number of bacilli. C Liver section $(10,000 \times$, Giemsa's stain). C1 Granular, vacuolated, and disintegrating liver cells. C2 Phagocytes in the walls of the portal capillary containing plague bacilli. D Section from the boundary zone of the kidney $(250 \times$, Giemsa's stain). D1 Congested vessels, one of which contained abundant plague bacilli. D2 Swollen basement membranes of tubules and capillaries. E Detection of Yersinia pestis in the sputum $(1000 \times$, eosin-methylene blue). E1 A single Yersinia pestis cell. E2 Yersinia pestis in chains (A-D are from Wu 1914; $\mathbf{E}$ is from Wu 1926). 
potential source of infection. To explore human-to-human transmission of the plague, the $Y$. pestis inhalation test was developed with a custom-designed apparatus to simulate natural infection through the respiratory tract in an animal model (Fig. 2). The results showed that $52.6 \%$ of droplets inoculated tarabagans were infected by $Y$. pestis, with a short incubation period (Wu and Eberson 1917; Wu and Jettmar 1934). Notably, early stages of the infection were more contagious, and death occurred an average of 4-6 days after infection. Moreover, tarabagans were susceptible to $Y$. pestis, and pneumonic plague could be transmitted from animal to animal through respiratory pathways. In 1921 and 1923, Dr. Wu Lien-Teh went twice to Russia and Mongolia again for expedition and found that bacteremia in rodents was a necessary factor in the spread of the plague in both wild and domesticated animals infected through both inhalation and flea bite (Wu 1924) (Fig. 2). In addition to acute and subacute cases, carriers may also harbor $Y$. pestis without symptoms. Furthermore, hibernation of wild rodents may promote the preservation of germ strains in the wild (Wu 1928). Thereafter, Dr. Wu Lien-Teh summarized his systemic studies and the incidence of the plague worldwide, with a particular emphasis in Russia, Mongolia, and China. From these results, he established the pneumonic plague theory, which, for the first time, demonstrated that the pneumonic plague was a particular type of plague. The pneumonic plague theory included information on the source of infection, route of transmission, susceptible population, and typical clinical manifestations and epidemiology. For example, Y. pestis could be isolated from samples from patients with pneumonic plague, and peeling and eating of infected animals could lead to periodic epidemics of acute or subacute bubonic plague in humans. Additionally, inhalation transmission was a main route for spread of pneumonic plague in a population, and disinfection and proper mask wearing may be effective strategies for clinical protection. $\mathrm{He}$ also concluded that pneumonic plague epidemics may be a secondary form of bubonic plague, whereas purely septicemic plague epidemics indicated the end of the epidemic period (Wu 1921; Wu 1922; Wu 1923; Wu 1926; Wu 1929).

\section{Establishment of the Earliest Pneumonic Plague Prevention and Control System Worldwide}

During the plague epidemic in 1910-1911, based on bacteriological identification and demonstration of the respiratory transmission route, $\mathrm{Dr}$. $\mathrm{Wu}$ Lien-Teh confirmed pneumonic plague as the cause of the epidemic and formulated epidemic prevention strategies, which were implemented successfully under the cooperation of multiple social parties (Fig. 3). The strategies included the following: (1) wearing a cotton mask correctly, environmental cleaning, disinfection and sterilization, and bathing for medical workers; (2) establishment of special hospitals with isolation wards and ambulances for pneumonic plague in order to provide local treatment in areas where epidemics occurred frequently; (3) zoning of the city, with the support of the army and police, to limit the flow of people; (4) strict control of rail traffic between Manzhouli and Harbin and inspection of other roads and frozen rivers along the bank; (5) burning of infected corpses and heavily contaminated residences; and (6) reporting of information on the epidemic every day to headquarters.
Fig. 2 In vivo inhalation experiment in tarabagans and the expedition study.

A Experimental model of Yersinia pestis inhalation in tarabagans. B Inhalation experiment in tarabagans on the spot (inside a tin-lined box). C Dr. Wu Lien-Teh taking the rectal temperature of a tarabagan in Manchouli. D The tarabagan. E The tarabagan flea. (A is from Wu et al. 1936; B is from Wu and Jettmar 1934; and C-E are from Wu 1913).
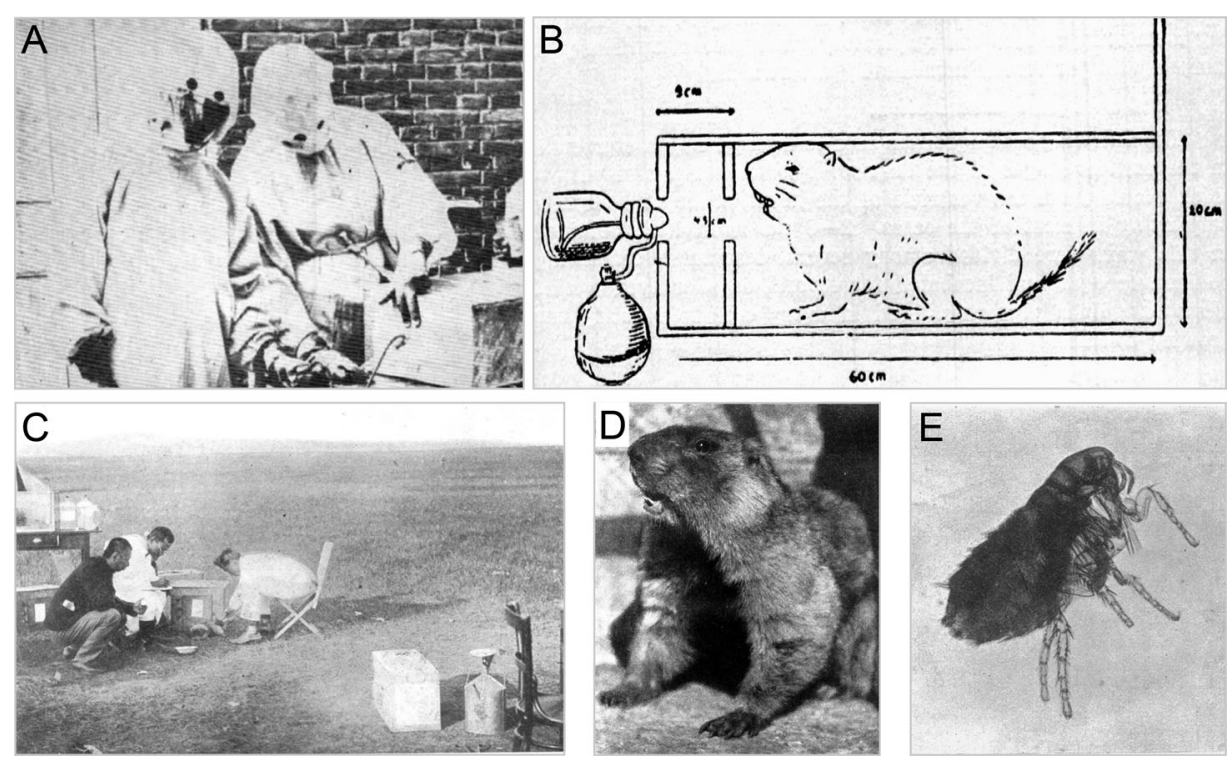

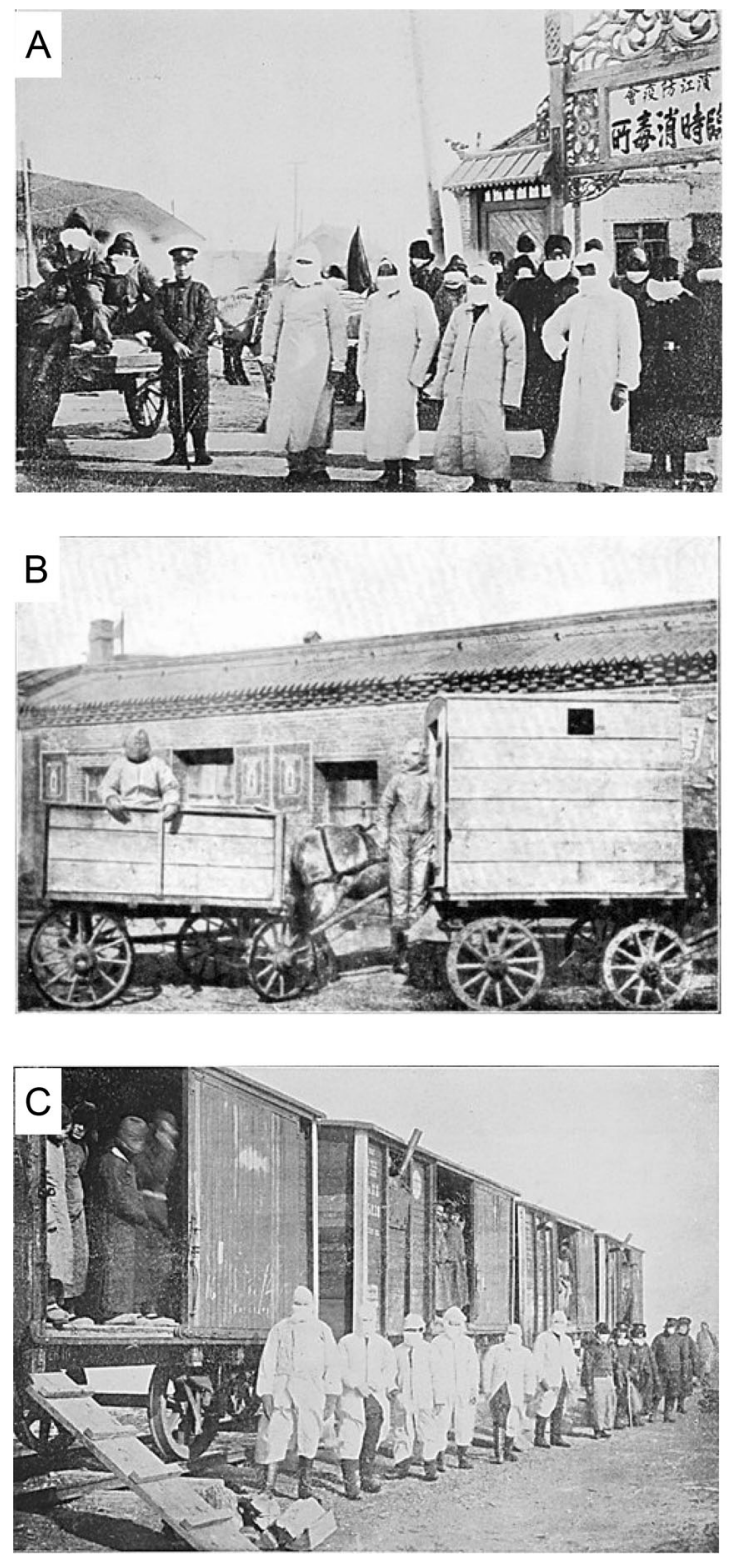
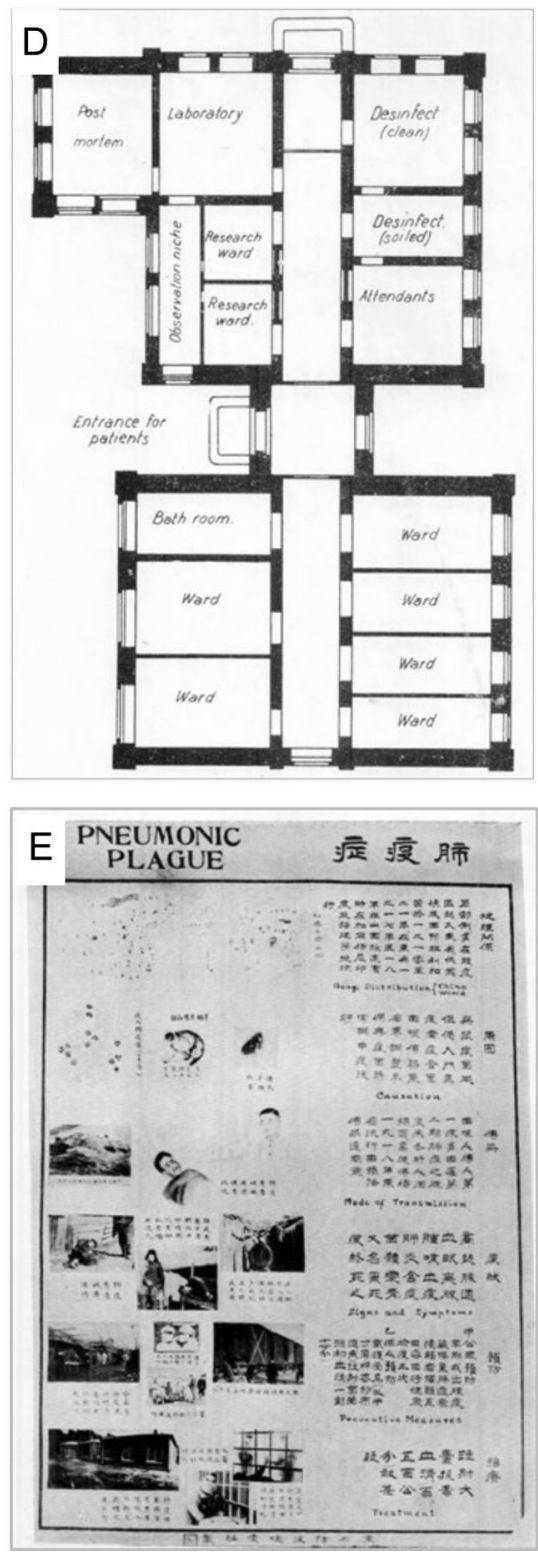

Fig. 3 Some measures in plague prevention and control. A Branch agencies of the North Manchurian Plague Prevention Service and epidemic prevention staff. B Improvised ambulance used in the 1911 epidemic in Harbin; the right one was used for sick individuals, and the left one was used for individuals who had come in contact with

In early March 1911, the epidemic outbreak was reported to be under control; the first reported day of zero deaths marked the initial stage of success. In 1912, in response to the potential regular resurgence of the plague epidemic, Dr. Wu Lien-Teh organized the establishment of the North Manchuria Plague Prevention Service headquartered in Harbin as a long-term plague control agency in the northeast China. Branches of this official agency were established in towns within the jurisdiction, including Taheiho, Sensing, Lahasusu, and Manzhouli. The agency and its offices were mainly responsible for epidemic sick individuals. C Plague isolation wards converted from train carriages. D The layout of a research ward for patients with plague in 1926. E. The flyer post for pneumonic plague, designed by Dr. Wu Lien-Teh (A-C are from Wu 1911; $\mathbf{D}$ is from Wu 1926; and $\mathbf{E}$ is from Wu et al. 1936).

prevention, general clinical work in affiliated hospitals, public health management, and assisting local officials and school teachers in organizing health courses and popular science education for children and the public (Wu LT and Wu Z 1918; Wu and Lin 1923; Wu 1959) (Fig. 3).

In 1920, Harbin was hit again by the bubonic plague rather than the pneumonic plague (Wu 1920). Owing to the accumulation of 10 years of work by the North Manchuria Plague Prevention Service, the spread of disease was successfully controlled within a short period. Afterward, Dr. $\mathrm{Wu}$ Lien-Teh elaborated on the geographic factors, clinical 
manifestations, and epidemic prevention in the affected areas, mainly focusing on significant issues, such as the condition of patients, contacts and suspected cases, protection of medical workers, ward management, refugee resettlement, medical expenses, and other issues (Wu 1923). The rapid eradication of the plague was thought to be dependent on the experience gained during the 1910-1911 epidemic and the cooperation of epidemic prevention agencies and systematic management, as well as constructive social powers (Wu et al. 1923). After additional developments, the North Manchurian Plague Prevention Service founded more standardized operating rooms, laboratories, libraries, museums, and other facilities. Special zones for the pneumonic plague were established in order to ensure the safety of medical staff (Fig. 3).

\section{Contributions of Dr. Wu Lien-Teh to the Development of the Modern Public Health System in China and the Effective Control of Emerging Infectious Diseases}

As the headquarters of epidemic prevention and control, the North Manchurian Plague Prevention Service holds positions of health supervision, scientific research, data integration, and hospital management. Based on these works, an efficient circulation system was developed from clinical sample collection to laboratory research and field trip investigations, to ultimately affect the application of disease prevention and control strategies. At the same time, relevant knowledge, technology, and a constructive epidemic prevention ideology were cultivated for other infectious diseases.

Dr. Wu Lien-Teh contributed greatly to the scientific cooperation and exchanges between China and foreign countries and the development of higher medical education in China. Based on his work in plague prevention, control, and studies, Dr. Wu Lien-Teh organized the International Plague Conference in Mukden (Shenyang), the first international scientific conference in Chinese history, and established the pneumonic plague theory. A Treatise of Pneumonic Plague was published by the League of Nations in 1926. In the same year, Harbin Medical College (Predecessor of Harbin Medical University) was founded, based on the North Manchurian Plague Prevention Service and its affiliated Harbin Hospital, to train new medical talents. In 1935, Dr. Wu Lien-Teh was nominated as a candidate for the Nobel Prize for "Work on pneumonic plague and especially the discovery of the role played by the Tarbagan in its transmission" (https://www.nobelprize. org/nomination/redirector/?redir=archive/). In addition, Dr. Wu Lien-Teh carried out prevention, control, and research work on other infectious diseases, such as the cholera epidemic in Harbin in 1919 and Shanghai in 1921 (Wu and Chun 1919; Wu and Chun 1920; Wu et al. 1926). He published monographs in both Chinese and English for the popularization of knowledge and medical guidance for medical workers against cholera (Wu et al. 1934). Additionally, Dr. Wu Lien-Teh's research work on infectious diseases also focused on the prevention and treatment of scarlet fever, tuberculosis, and sexually transmitted diseases (Wu 1934a; Wu 1927). Moreover, Dr. Wu Lien-Teh was one of the founders of the Chinese Medical Association and the National Medical Journal of China (Wu 1915). He wrote the History of Chinese Medicine to present the medical experience of China to the world (Wong and $\mathrm{Wu}$ 1932). He introduced the scientific concept of health and quarantine to China and led the establishment of the National Quarantine Service in Shanghai in 1930 (Wu 1934b), defending China's national sovereignty and health security. His academic thought has not only been applied to medical work in China but also contributed greatly to the improvement of the epidemic prevention system worldwide (Fig. 4).

Currently, we are encountering the COVID-19 pandemic outbreak worldwide. The causative pathogen of COVID-19 was identified quickly, and the clinical characteristics and transmission route of COVID-19 were demonstrated (Zhu et al. 2020; Tan et al. 2020; The 2019-nCoV Outbreak Joint Field Epidemiology Investigation Team and Li 2020; Li et al. 2020). Based on the route of transmission of COVID-19, which mainly involves the respiratory tract, it was suggested that citizens should wear masks, keep a reasonable social distance, and wash their hands frequently to prevent human-to-human transmission (Jiang et al. 2020). Pathological autopsy was performed to determine the damage to tissues and organs caused by the coronavirus infection (Xu et al. 2020). Importantly, longterm research on coronavirus and investigation of the natural viral host after the SARS epidemic also helped us identify wild bats as the most probable natural origin of 2019-nCoV (Zhou et al. 2020). In vivo experiments with this virus were performed to identify the intermediate animal hosts between wild bats and humans (Shi et al. 2020). In order to effectively combat the outbreak of COVID-19 in patients and reduce the risk of virus spread between infected persons and healthy population, specialized facilities, such as intensive care units, general care wards, and specialized surgery areas, were set up in special hospitals ( $\mathrm{Li}$ et al. 2020). Mobile cabin hospitals and special locations were established for isolation, observation, and treatment of patients and contacted groups. International, domestic, and local transportation methods were temporarily restricted to limit the frequency of person-to-person contact. Furthermore, modern communication tools, such as smart phones, were used to record the 


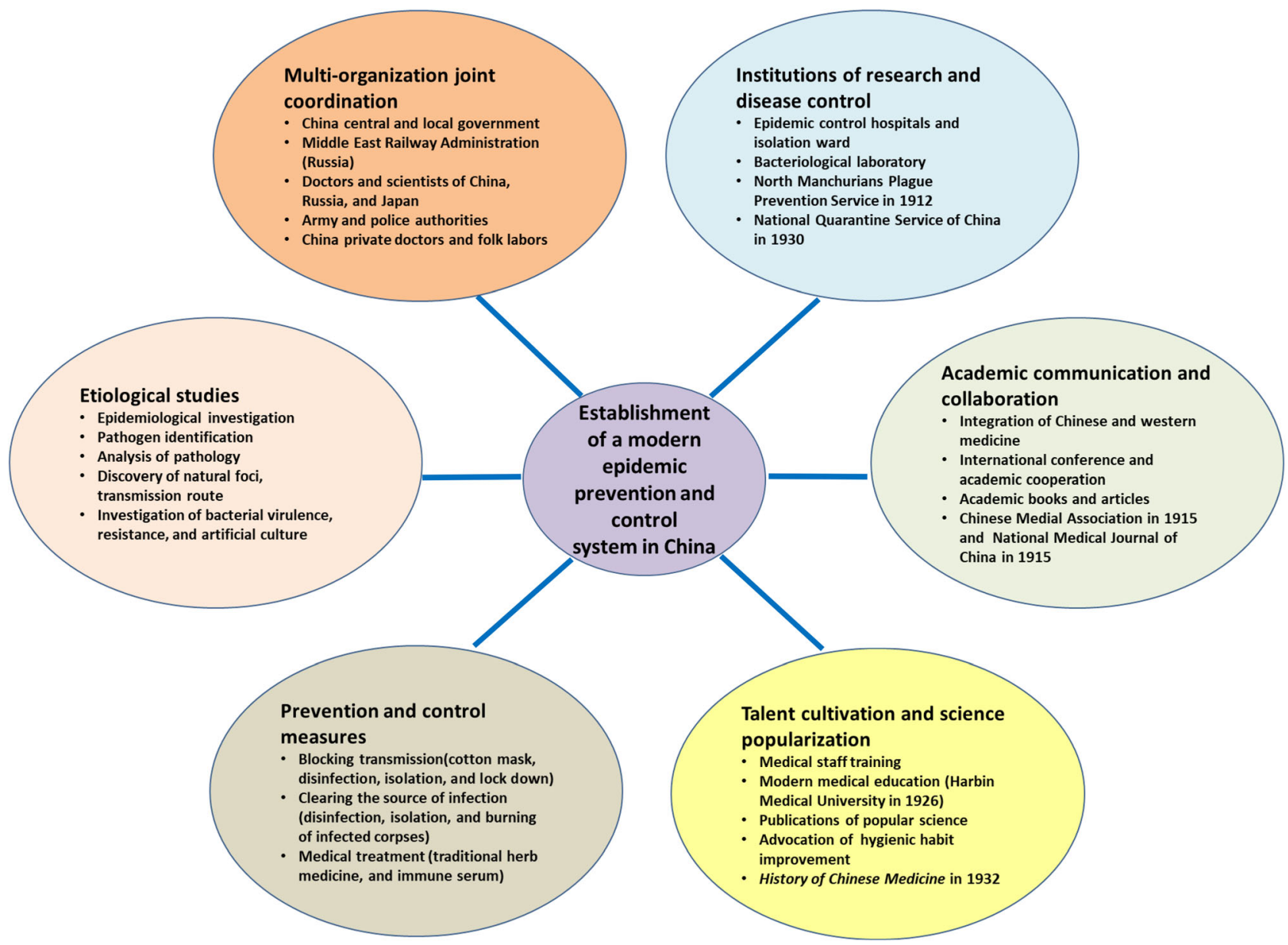

Fig. 4 Dr. Wu Lien-Teh's contribution to the development of a modern epidemic prevention system in China.

details of population flow as an efficient approach to deal with the risk of latent viral spread and determine the contact history of infected individuals.

Compared with the epidemic prevention system established by Dr. Wu Lien-Teh 110 years ago, the system for the prevention and control of emerging infectious diseases in China and worldwide is more complete and effective today. However, his successful experience and outstanding contribution into the antiplague campaign during the pneumonic plague still inspire us to face the challenges of emerging infectious diseases, such as the COVID-19 pandemic, in a more globalized economy. More efficient and effective national and international response systems and talent teams should be established to increase global capacity to respond to emerging infectious diseases. A worldwide consensus on jointly combating emerging infectious diseases is necessary for the fundamental guarantee of public health and the future of mankind.

Today, we commemorate Dr. Wu Lien-Teh's 140th birthday and honor his great contributions to the establishment of modern medical and public health systems in
China. Despite these accomplishments and advancements in health care, we have recently experienced a pneumonic plague event in Inner Mongolia and Beijing (Wang et al. 2019) and are still combatting COVID-19. More importantly, we face even more challenges of emerged and emerging pathogens (Gao 2018). Dr. Wu Lien-Teh's legacy must be continued. Therefore, we propose that research groups around the world should team up to develop improved approaches for combatting infectious diseases.

Acknowledgements This work was supported by Dr. Wu Lien-Teh Scientific Funds of Harbin Medical University.

\section{Compliance with Ethics Standards}

Conflict of interest The authors declare that they have no conflict of interest.

Animal and Human Rights Statement This article does not contain any studies with human or animal subjects performed by any of the authors. 


\section{References}

Gao GF (2018) From “A” IV to "Z" IKV: attacks from emerging and re-emerging pathogens (Commentary). Cell 172:1157-1159

Jiang X, Wang H, Gao GF, Jiang X (2020) Wearing face masks-the simple and effective way to block the infection source of COVID-19. China CDC Wkly 2:268-269

Li Q, Guan X, Wu P, Wang X, Zhou L, Tong Y, Ren R, Leung KSM, Lau EHY, Wong JY, Xing X, Xiang N, Wu Y, Li C, Chen Q, Li D, Liu T, Zhao J, Liu M, Tu W, Chen C, Jin L, Yang R, Wang Q, Zhou S, Wang R, Liu H, Luo Y, Liu Y, Shao G, Li H, Tao Z, Yang Y, Deng Z, Liu B, Ma Z, Zhang Y, Shi G, Lam TTY, Wu JT, Gao GF, Cowling BJ, Yang B, Leung GM, Feng Z (2020a) Early transmission dynamics in Wuhan, China, of novel coronavirus-infected pneumonia. N Engl J Med 382:1199-1207

Li Z, Chen Q, Feng L, Rodewald L, Xia Y, Yu H, Zhang R, An Z, Yin W, Chen W, Qin Y, Peng Z, Zhang T, Ni D, Cui J, Wang Q, Yang X, Zhang M, Ren X, Wu D, Sun X, Li Y, Zhou L, Qi X, Song T, Gao GF, Feng Z, China CDC (2020b) Active case finding with case management: the key to tackling the COVID19 Pandemic. Lancet 396:63-70

Reperant LA, Osterhaus A (2017) AIDS, Avian flu, SARS, MERS, Ebola, Zika... what next? Vaccine 35:4470-4474

Shi J, Wen Z, Zhong G, Yang H, Wang C, Huang B, Liu R, He X, Shuai L, Sun Z, Zhao Y, Liu P, Liang L, Cui P, Wang J, Zhang X, Guan Y, Tan W, Wu G, Chen H, Bu Z (2020) Susceptibility of ferrets, cats, dogs, and other domesticated animals to SARScoronavirus 2. Science 368:1016-1020

Strong RP (1912) Report of the international plague conference. Bureau of Publishing, Manila

Tan W, Zhao X, Ma X, Wang W, Niu P, Xu W, Gao GF, Wu G (2020) A novel coronavirus genome identified in a cluster of pneumonia cases-Wuhan, China 2019-2020. China CDC Wkly 2:61-62

The 2019-nCoV Outbreak Joint Field Epidemiology Investigation Team, Li Q (2020) An outbreak of NCIP (2019-nCoV) infection in China-Wuhan, Hubei Province, 2019-2020. China CDC Wkly 2:79-80

Wang Y, Zhou L, Fan M, Wang Q, Li J, Li Q, Feng Z, Gao GF, Xu C, Chen L, Li W, Zhang Y (2019) Isolated cases of plague-inner Mongolia-Beijing, 2019. China CDC Wkly 1:13-16

Wong KC, Wu LT (1932) History of Chinese Medicine. The Tientsin Press Ltd., Tientsin China

Wu LT (1911) Views of Harbin Fuchiatien taken during the plague epidemic December 1910-March 1911. The Commercial Press Ltd, Shanghai

Wu LT (1913a) First report of The North Manchurian Plague Prevention Service. J Hyg 13:237-301

Wu LT (1913b) Investigations into the relationship of the tarabagan (Mongolian marmot) to a plague. Lancet 182:529-535

Wu LT (1914) Note on the histology of some of the lesions found in pneumonic plague. J Path Bacteriol 19:1-32

Wu LT (1915) The National Medical Association of China. Natl Med J China 29:406-408

Wu LT (1918) The virulence of plague. Natl Med J China 4:135-140

Wu LT (1920) Winter outbreak of bubonic plague in Manchuria. Natl Med J China 6:225-226

Wu LT (1921) The relationship between the plague of the far east and the epidemic in North Manchuria. Natl Med J China 7:197-211

Wu LT (1922) Plague in the orient with particular reference to the Manchurian outbreak. J Hyg 21:62-76
Wu LT (1923) The second pneumonic plague epidemic in Manchuria, 1920-1921. J Hyg 21:262-288

Wu LT (1924) A further note on natural and experimental plague in tarabagans. J Hyg 22:329-334

Wu LT (1926) A treatise of pneumonic disease. League of Nations, Health Organization

Wu LT (1927) The problem of venereal diseases in China. Natl Med J China 41:28-36

Wu LT (1928) The perpetuation of plague among wild rodents. Am J Hyg 8:649-670

Wu LT (1929) An overview of plague prevalence and prevention. National Medical Journal of China 15:217-250

Wu LT (1934a) Tuberculosis. Natl Med J China 20:65-97

Wu LT (1934b) National quarantine service reports, memorial volume. The Mercury Press, Shanghai, China

Wu LT (1959) Plague fighter: the autobiography of a modern Chinese physician. W. Heffer \& Sons Ltd., Cambridge

Wu L-T, Chun JWH (1919) The recent cholera epidemic in China. Natl Med J China 5:182-198

Wu L-T, Chun JWH (1920) The management of the 1919 cholera epidemic in Harbin. Natl Med J China 6:4-16

Wu LT, Eberson F (1917) Transmission of pulmonary and septicemic plague among marmots. J Hyg 16:1-11

Wu LT, Jettmar HM (1926) Notes on the vitality of plague bacilli in stained smears. Natl Med J China 12:1-8

Wu LT, Jettmar HM (1934) A systematic experimental study of the pathology of pneumonic plague in the tarabagan and sisel (Sulik). North Manchurian Plague Prevention Service Report 1912-1932, memorial volume, pp 163-188. The Mercury Press, Shanghai

Wu LT, Lin J (1923) The relationship between the plague of the far east and the epidemic in North Manchuria. Natl Med J China 8:97-109

Wu LT, Wu Z (1918) Plague and disinfection. Broad Med Rep 3:54-65

Wu LT, Chun WH, Pollitzer R (1923) Plague in Manchurian. J Hyg 21:307-358

Wu LT, Chun WH, Pollitzer R (1926) Preliminary report on the cholera epidemic of 1926. Natl Med J China 12:413-452

Wu LT, Chun JWH, Pollitzer R, Wu CY (1934) Cholera (A manual for the medical profession in China). National Quarantine Service, Shanghai, China

Wu LT, Chun JWH, Pollitzer R, Wu CY (1936) Plague (A manual for medical and public health workers). National Quarantine Service, Shanghai Station

Xu Z, Shi L, Wang Y, Zhang J, Huang L, Zhang C, Liu S, Zhao P, Liu H, Zhu L, Tai Y, Bai C, Gao T, Song J, Xia Peng, Dong J, Zhao J, Wang FS (2020) Pathological findings of COVID-19 associated with acute respiratory distress syndrome. Lancet Respir Med 8:420-422

Zhou P, Yang XL, Wang XG, Hu B, Zhang L, Zhang W, Si HR, Zhu Y, Li B, Huang CL, Chen HD, Chen J, Luo Y, Guo H, Jiang RD, Liu MQ, Chen Y, Shen XR, Wang X, Zheng XS, Zhao K, Che QJ, Deng F, Liu LL, Yan B, Zhan FX, Wang YY, Xiao GF, Shi ZL (2020) A pneumonia outbreak associated with a new coronavirus of probable bat origin. Nature 579:270-273

Zhu N, Zhang D, Wang W, Li X, Yang B, Song J, Zhao X, Huang B, Shi W, Lu R, Niu P, Zhan F, Ma X, Wang D, Xu W, Wu G, Gao GF, Tan W, China Novel Coronavirus Investigating and Research Team (2020) A novel coronavirus from patients with pneumonia in China, 2019. N Engl J Med 382:727-733 